\title{
As ações de educação alimentar e nutricional e o nutricionista no âmbito do Programa Nacional de Alimentação Escolar
}

\author{
Food and nutrition education actions and the nutritionist \\ within the scope of the National School Food Program
}

Simoni Urbano da Silva ${ }^{1}$

Estelamaris Tronco Monego ${ }^{1}$

Lucilene Maria de Sousa ${ }^{1}$

Géssica Mercia de Almeida ${ }^{1}$

${ }^{1}$ Faculdade de Nutrição, Universidade Federal de Goiás. Rua 227 Qd. 68/s/nº, Setor Leste Universitário. 74605-080 Goiânia GO Brasil.

simoni.urbano@gmail.com
Abstract The National School Food Program (NSFP) recommends the promotion of healthy eating habits by offering meals along with food and nutrition education (FNE) actions. The objective of this study was to identify the FNE in the NSFP and its relationship with the nutritionist's profile. It involved an analytical, cross-sectional study conducted in cities in the state of of Goiás, Brazil. Data was collected by telephone, and the association between the outcome variable (achievement of FNE actions) and exposure (school population and nutritionist performance) was done by Pearson's chi-square test of with a 5\% significance level. In this study, 214 (87\%) municipalities were included, among which, $91.1 \%$ promote FNE actions with predominantly biannual frequency $(25.3 \%$ in schools and $23.2 \%$ in day care centers). The nutritionist presence was associated to the FNE ( $p=0.001)$. FNE actions are performed in most municipalities, however with low frequency. The association indicates the relevance of a nutritionist present in conditions that heed the legislation, enabling the performance of their duties, as defined by the Legal Framework of the NSFP and the Federal Council of Nutritionists. Key words Food and nutrition education, School food, Nutritionist, Health promotion, School health
Resumo O Programa Nacional de Alimentação Escolar (PNAE) preconiza a promoção de práticas alimentares saudáveis por meio da oferta de refeições juntamente com ações de educação alimentar e nutricional (EAN). O objetivo deste trabalho foi identificar as ações de EAN no PNAE e sua relação com o perfil do nutricionista. Estudo transversal analítico, realizado em municípios de Goiás, Brasil. Dados coletados por telefone, sendo a associação entre a variável desfecho (realização de EAN) $e$ as de exposição (população escolar e atuação do nutricionista) feita por teste qui-quadrado de Pearson, nível de significância de 5,0\%. Foram incluidos 214 (87\%) municipios, dos quais 91,1\% promovem EAN, com frequência predominantemente semestral (25,3\% nas escolas e 23,2\% nas creches). A presença do nutricionista mostrou-se associada à EAN ( $p=0,001)$. Ações de EAN são executadas na maioria dos municípios, entretanto com baixa frequência. A associação encontrada indica a relevância da presença do nutricionista, em condições que atendam à legislação, possibilitando o cumprimento de suas atribuições, conforme definido pelo Marco Legal do PNAE e do Conselho Federal de Nutricionistas.

Palavras-chave Educação alimentar e nutricional, Alimentação escolar, Nutricionista, Promoção da saúde, Saúde escolar 


\section{Introdução}

O Programa Nacional de Alimentação Escolar (PNAE) é política de Estado vinculada ao Fundo Nacional de Desenvolvimento da Educação do Ministério da Educação (FNDE/MEC). Sua origem data de 1955, sendo considerado um dos maiores programas de alimentação escolar do mundo, atendendo de forma gratuita aos alunos matriculados na educação básica de escolas públicas, filantrópicas e comunitárias de todos os municípios do país ${ }^{1-3}$.

A legislação vigente identifica como objetivo do PNAE contribuir para o crescimento e o desenvolvimento biopsicossocial, o rendimento escolar, a aprendizagem e a formação de práticas alimentares saudáveis dos alunos, por meio da realização de ações de educação alimentar e nutricional (EAN) e da oferta de refeições que garantam as necessidades nutricionais dos estudantes no período em que estes se encontram na escola $^{2,3}$.

Embora o potencial educativo da alimentação escolar tenha sido discutido já na década de 1970, pelo extinto Instituto Nacional de Alimentação e Nutrição ${ }^{4}$, a inclusão da EAN no PNAE se deu apenas em 2006, com a Portaria Interministerial $n^{\circ} 1.010^{5,6}$. Inicialmente concebida como uma diretriz do Programa, com a promulgação da Lei no 11.947, de 16 de junho de 2009, a EAN se tornou eixo prioritário do PNAE, sendo considerada como avanço na execução do mesmo ${ }^{2}$.

No âmbito do PNAE, a EAN representa $o$ conjunto de ações formativas, de prática contínua e permanente, transdisciplinar, intersetorial e multiprofissional, que objetiva estimular a adoção voluntária de práticas e escolhas alimentares saudáveis que colaborem para a aprendizagem, o estado de saúde do escolar e a qualidade de vida do indivíduo. Nesta perspectiva, são pressupostos das ações de EAN a promoção da oferta de alimentação saudável e adequada na escola; atividades que favoreçam hábitos alimentares regionais e culturais; ações que articulem políticas em todos os níveis, no âmbito da alimentação escolar; utilização de metodologias inovadoras para o trabalho pedagógico e desenvolvimento de tecnologias sociais voltadas para a alimentação escolar; além da utilização do alimento como ferramenta pedagógica ${ }^{3}$.

Por esta razão, a alimentação escolar assume um caráter pedagógico, devendo estimular a integração de temas relativos à alimentação e nutrição no currículo escolar, de modo transversal. Assim, supõe-se que o Programa represente um espaço para o desenvolvimento de atividades de promoção da saúde, construção de conhecimentos e de aprendizagem na escola com vistas à segurança alimentar e nutricional dos estudantes ${ }^{7}$.

O nutricionista, profissional regulamentado pela Lei no 8.234, de 17 de setembro de 1991 é o responsável técnico (RT) pela execução do PNAE $^{2,8}$. De acordo com o Conselho Federal de Nutricionistas (CFN), além do RT, poderão integrar o quadro técnico (QT) do PNAE outros nutricionistas habilitados, aos quais competem, dentre outras atribuições, coordenar e realizar ações de EAN, de forma articulada com a direção e coordenação pedagógica das escolas, em uma perspectiva multiprofissional ${ }^{3,9}$, considerando a legitimidade dos saberes de diferentes naturezas.

Apesar da legislação favorável, as ações de EAN ainda são pouco presentes em escolas atendidas pelo PNAE nos municípios brasileiros. Das 568 prefeituras avaliadas em 2011 pelo Prêmio Gestor Eficiente da Merenda Escolar, 51\% incluíram o tema EAN no currículo escolar; 49\% utilizavam a formação da comunidade escolar como ação de EAN e 40\% realizavam oficinas culinárias, enquanto na região Centro-Oeste, as proporções observadas foram de $33 \%$, 50\% e $40 \%$, respectivamente ${ }^{10}$.

Este estudo tem por objetivo identificar e caracterizar as ações de EAN executadas no âmbito do PNAE no estado de Goiás, e analisar as possíveis relações com o perfil do nutricionista. Desta forma, pretende-se contribuir para uma maior compreensão do papel deste profissional no contexto da alimentação escolar, visando ampliar o potencial das ações de EAN.

\section{Métodos}

Estudo transversal analítico, executado pelo Centro Colaborador em Alimentação e Nutrição do Escolar da Universidade Federal de Goiás (CECANE UFG) e financiado pelo Fundo Nacional de Desenvolvimento da Educação do Ministério da Educação (FNDE/MEC).

A amostra incluiu todos os municípios de Goiás no ano de 2012 ( $\mathrm{n}=246)$. Destes, foram excluídos aqueles que não recebiam recurso do PNAE, além dos municípios onde os representantes locais do PNAE não souberam responder sobre as ações de EAN. Além disso, foram considerados como perdas os municípios onde houve recusa ou insucesso de contato telefônico após 10 tentativas em diferentes dias e horários, sendo as sete primeiras direcionadas ao nutricionista RT. 
A coleta dos dados foi realizada no período de abril a agosto de 2012, por sete entrevistadores (duas nutricionistas e cinco estudantes de $\mathrm{Nu}$ trição), devidamente treinados. As informações foram obtidas por meio telefônico, utilizando-se o software Skype, e registradas em formulário estruturado.

As entrevistas foram direcionadas prioritariamente ao nutricionista RT do PNAE. Entretanto, em municípios que não dispunham deste profissional, ou naqueles em que o nutricionista não foi encontrado após sete tentativas, a entrevista foi realizada com o gestor local do programa (coordenador, gerente ou diretor de merenda/ alimentação escolar, Secretário de Educação ou outro profissional que se declarasse responsável pela gestão do PNAE no município). A inclusão de ambas categorias objetivou a caracterização das ações de EAN frente à presença ou não de nutricionista RT do PNAE.

$\mathrm{O}$ questionário foi estruturado em quatro blocos: identificação do município (aplicado a nutricionistas ou gestores do PNAE); informações sobre a atuação do nutricionista RT (aplicado somente a estes profissionais); informações sobre as ações de EAN; e investigação quanto a limitações e facilidades para a realização de ações de EAN, destinado aos municípios onde estas ações não são executadas (os dois últimos aplicados a nutricionistas ou gestores do PNAE).

Para a elaboração do questionário foram consideradas as informações obtidas em levantamento bibliográfico sobre o tema da pesquisa, além de informações sobre a atuação dos nutricionistas, coletadas durante a execução de projetos de pesquisa e/ou extensão realizados pelo CECANE UFG. O instrumento foi avaliado por um grupo de nove professores da UFG e Universidade Federal de Santa Catarina, com experiência em coleta de dados por entrevista telefônica e/ou projetos que envolviam o ambiente escolar.

Para o controle de qualidade e armazenamento do consentimento dos entrevistados, as entrevistas foram gravadas por meio do software livre MP3 Skype Recorder, sendo ouvidas por dois supervisores e comparadas aos questionários preenchidos. Quando necessário, correções foram realizadas.

Foram analisadas as seguintes variáveis: população escolar dividida em quartis $(\mathrm{Q} 1$ - até 452 estudantes; Q2 - de 453 a 879; Q3 - de 880 a 1830; Q4 - 1831 ou mais); presença do nutricionista RT; presença de outro(s) nutricionista(s) no QT do PNAE; atuação do nutricionista como RT da alimentação escolar em mais de um mu- nicípio; tipo de vínculo empregatício; tempo de atuação na alimentação escolar do município; carga horária semanal de trabalho; dedicação a outros setores da prefeitura; e frequência de atuação na alimentação escolar. Avaliou-se ainda a realização de ações de EAN nos municípios; o responsável pela coordenação e execução das ações de EAN; as formas como escolas e creches são orientadas a realizar ações de EAN; os tipos de ações de EAN executadas e a inserção da EAN no projeto político pedagógico (PPP).

Os dados foram digitados duplamente no software Epi Info versão 3.5.2 e analisados com auxílio do aplicativo Stata S/E 12. A análise foi realizada de forma descritiva, com resultados expressos em percentuais, incluindo somente as respostas válidas. Para verificar a associação entre a variável desfecho (realização de ações de EAN) e as de exposição (população escolar e características da atuação do nutricionista), utilizou-se o teste qui quadrado de Pearson, sendo as diferenças consideradas a um nível de significância de 5,0\%.

$\mathrm{O}$ estudo atendeu ao preconizado pela Resolução do Conselho Nacional de Saúde $\mathrm{n}^{\circ}$ 196/96, vigente na época da coleta de dados, e foi aprovado pelo Comitê de Ética em Pesquisa da UFG. Por se tratar de entrevista telefônica, o consentimento livre e esclarecido foi substituído pela gravação do consentimento verbal obtido no contato telefônico com os entrevistados.

\section{Resultados}

Participaram deste estudo 214 municípios, o que representa $87 \%$ da população estudada. Houve recusa de 11 (4,5\%) entrevistados; impossibilidade de contato com o representante do PNAE em $15(6,1 \%)$ municípios e exclusão por repasse do recurso do PNAE suspenso ou questionários não respondidos em seis municípios $(2,4 \%)$.

Dos municípios incluídos, 199 (93,0\%) possuíam nutricionista RT do PNAE, e em 178 $(83,2 \%)$ este profissional foi localizado e entrevistado. Destes, em apenas 11 (5,1\%) foi relatada a presença de outros nutricionistas no QT do PNAE. Ressalta-se que, dentre os nutricionistas entrevistados, 23 (12,9\%) trabalhavam como RT do PNAE em outro(s) município(s) além daquele que era alvo da entrevista, sendo 17 (9,5\%) RT em dois municípios e seis $(3,4 \%)$ em três municípios.

Na Tabela 1 são apresentadas as características de atuação dos nutricionistas entrevistados no estudo. A maioria relatou vínculo empregatício 
Tabela 1. Caracterização dos nutricionistas participantes do estudo "As ações de educação alimentar e nutricional e o nutricionista no âmbito do Programa Nacional de Alimentação Escolar”. Goiás, $2012\left(\mathrm{n}=178^{\star}\right)$.

\begin{tabular}{lrr}
\hline \multirow{2}{*}{ Variáveis coletadas } & \multicolumn{2}{c}{ Frequência } \\
\cline { 2 - 3 } & $\mathrm{n}$ & \multicolumn{1}{c}{$\%$} \\
\hline Tipo de vínculo empregatício com a & & \\
prefeitura & & \\
Contrato de prestação de serviço & 127 & 71,3 \\
Concurso público & 40 & 22,5 \\
Cargo em comissão & 8 & 4,5 \\
Profissional autônomo & 2 & 1,1 \\
Não sabe & 1 & 0,6 \\
Carga horária semanal contratada & & \\
1 a 9 horas & 25 & 14,0 \\
10 a 19 horas & 18 & 10,1 \\
20 a 29 horas & 47 & 26,4 \\
30 a 44 horas & 84 & 47,2 \\
Outras cargas horárias & 4 & 2,2 \\
Frequência de atuação na alimentação & & \\
escolar & & \\
4 a 5 vezes/semana & 68 & 38,2 \\
3 vezes/semana & 25 & 14,1 \\
2 vezes/semana & 39 & 21,9 \\
1 vez/semana & 46 & 25,8 \\
Tempo de atuação na alimentação & & \\
escolar do município & & \\
Até 1 ano & 63 & 35,4 \\
1 a 2 anos & 41 & 23,0 \\
2 a 5 anos & 47 & 26,4 \\
> 10 anos 10 anos & 26 & 14,6 \\
& 1 & 0,6 \\
\hline
\end{tabular}

${ }^{*}$ Municípios onde o entrevistado foi o nutricionista.

com a prefeitura por meio de contrato de prestação de serviço $(\mathrm{n}=127 ; 71,3 \%)$ e carga horária inferior a 30 horas por semana $(\mathrm{n}=90 ; 50,6 \%)$. Além dessas informações, $56(31,4 \%)$ indicaram acumular outras atividades além da alimentação escolar no município, sendo $45(80,4 \%)$ na área da saúde e sete $(12,5 \%)$ na saúde e assistência social. Outros setores, como padarias, asilos e restaurantes municipais também foram citados ( $\mathrm{n}$ $=4 ; 7,1 \%)$.

Sobre as ações de EAN, observou-se que em $195(91,1 \%)$ municípios as mesmas eram realizadas nas escolas e creches atendidas pelo PNAE. Dentre estes, em 194 (99,5\%) elas aconteciam nas escolas e em $142(72,8 \%)$ nas creches. Considerando as escolas e as creches, a frequência de realização de ações de EAN mais referida foi a semestral ( $\mathrm{n}=49 ; 25,26 \%$ e $\mathrm{n}=33 ; 23,2 \%$ ), seguida pela mensal $(\mathrm{n}=39,20,1 \%$ e $\mathrm{n}=24,17,0 \%)$, trimestral $(\mathrm{n}=24,12,4 \%$ e $\mathrm{n}=17,12,0 \%)$, bimestral $(\mathrm{n}=16,8,2 \%$ e $\mathrm{n}=12,8,4 \%)$ e anual $(\mathrm{n}=14$, $7,2 \%$ e $\mathrm{n}=10,7,0 \%)$.

Ainda considerando os municípios em que as ações de EAN são realizadas, cerca de $90 \%$ dos entrevistados relataram haver um responsável por sua coordenação, com ênfase no nutricionista RT $(60,2 \%)$. Dentre os responsáveis pela execução destas ações, o nutricionista RT ( $\mathrm{n}=147$; $79,0 \%)$ e os professores $(\mathrm{n}=127 ; 68,3 \%)$ foram os mais citados (Tabela 2).

Do total de municípios cujos entrevistados afirmaram realizar ações de EAN, em 166 $(85,1 \%)$ as escolas e as creches recebiam orientações para esta atividade. Reuniões pedagógicas (n $=52 ; 31,3 \%)$ foram as mais citadas. Dos entrevistados, $175(81,0 \%)$ souberam relatar quais os tipos de ações desenvolvidos junto aos estudantes, com destaque para a realização de palestras $(\mathrm{n}=$ $143 ; 81,7 \%)$ e atividades lúdicas ( $\mathrm{n}=87 ; 54,3 \%$ ) (Tabela 2).

Dentre os entrevistados que relataram realizar atividades de EAN, 119 (61,0\%) informaram que as ações estão inseridas no PPP das unidades escolares, porém apenas $67(56,3 \%)$ souberam relatar em quais disciplinas o tema está inserido. Dos que informaram, a disciplina de Ciências foi a mais citada $(\mathrm{n}=59 ; 88,1 \%)$, seguida por Língua Portuguesa $(\mathrm{n}=33 ; 49,2 \%)$ e Matemática $(\mathrm{n}=$ 27; 40,3\%).

Dos 19 municípios em que as ações de EAN não eram executadas, em 18 (94,7\%) o entrevistado soube relatar os motivos para esta não realização, sendo os mais citados a ausência de nutricionista $(33,3 \%)$, a falta de tempo $(33,3 \%)$, o desinteresse da comunidade escolar $(22,2 \%)$ e a insuficiência de recursos humanos (16,7\%).

Foi encontrada associação positiva entre a presença do nutricionista e a realização de ações de EAN $(p=0,001)$. Não houve associação entre as demais características de atuação dos nutricionistas ou população escolar e a realização de ações de EAN (Tabela 3).

\section{Discussão}

Esta pesquisa revela, de forma inédita, a situação da EAN no âmbito do PNAE nos municípios de Goiás quanto a alguns aspectos de identificação de sua execução e em relação à atuação do nutricionista, profissional que possui a atribuição legal de responsabilidade técnica pela alimentação escolar. 
Tabela 2. Caracterização das ações de educação alimentar e nutricional realizadas no âmbito do Programa Nacional de Alimentação Escolar em municípios goianos. Goiás, 2012.

\begin{tabular}{|c|c|c|}
\hline \multirow{2}{*}{ Variáveis analisadas } & \multicolumn{2}{|c|}{ Frequência } \\
\hline & $\mathbf{n}$ & $\%$ \\
\hline \multicolumn{3}{|l|}{ Responsável pela coordenação das ações de EAN $\left(n=176^{1}\right)^{*}$} \\
\hline Nutricionista RT & 106 & 60,2 \\
\hline Diretor da escola & 46 & 26,1 \\
\hline Coordenador pedagógico & 46 & 26,1 \\
\hline Gestor local do PNAE & 39 & 22,2 \\
\hline Professores & 23 & 13,1 \\
\hline Secretário de Educação & 10 & 4,6 \\
\hline $\begin{array}{l}\text { Outros atores (Conselho de Alimentação Escolar, manipulador de alimentos, } \\
\text { secretários das escolas, dentre outros) }\end{array}$ & 14 & 7,9 \\
\hline \multicolumn{3}{|l|}{ Responsável pela execução das ações de EAN $\left(n=186^{2}\right)^{\star}$} \\
\hline Nutricionista RT & 147 & 79,0 \\
\hline Professores & 127 & 68,3 \\
\hline Coordenador pedagógico & 28 & 15,0 \\
\hline Gestor local do PNAE & 25 & 13,4 \\
\hline Diretor das escolas & 13 & 7,0 \\
\hline Manipulador de alimentos & 10 & 5,4 \\
\hline $\begin{array}{l}\text { Outros atores (estudantes, profissionais de saúde, Secretário de Educação, } \\
\text { Conselho de Alimentação Escolar, dentre outros) }\end{array}$ & 30 & 16,1 \\
\hline \multicolumn{3}{|l|}{ Formas de orientação para as escolas e creches realizarem EAN $\left(n=166^{3}\right)^{*}$} \\
\hline Reuniões pedagógicas ou com comunidade escolar** & 52 & 31,3 \\
\hline Formação específica para professores e/ou coordenadores & 24 & 14,4 \\
\hline Orientações durante visitas técnicas & 20 & 12,0 \\
\hline Informativos para diretores, coordenadores e professores & 13 & 7,8 \\
\hline Formação de multiplicadores (não especificados) & 11 & 6,6 \\
\hline Inserção de projetos no calendário escolar & 11 & 6,6 \\
\hline Outras formas de orientação & 17 & 10,2 \\
\hline Não sabe & 6 & 3,6 \\
\hline \multicolumn{3}{|l|}{ Ações de EAN realizadas $\left(\mathrm{n}=175^{4}\right)^{*}$} \\
\hline Palestras & 143 & 81,7 \\
\hline Atividades lúdicas (jogos, teatros, brincadeiras) & 87 & 49,7 \\
\hline Eventos como Feira de Ciências ou Semana da Alimentação & 57 & 32,6 \\
\hline Hortas escolares & 29 & 16,6 \\
\hline Oficinas de arte culinária & 27 & 15,4 \\
\hline Utilização de música, vídeos e/ou filmes & 24 & 13,7 \\
\hline Reuniões, palestras ou informativos direcionados aos pais ${ }^{\star * *}$ & 19 & 10,9 \\
\hline Murais educativos e/ou cartazes & 18 & 10,3 \\
\hline Atividades pedagógicas inseridas no currículo escolar & 16 & 9,1 \\
\hline Formação da comunidade escolar ${ }^{* *}$ & 15 & 8,6 \\
\hline Oficinas de alimentação saudável & 14 & 8,0 \\
\hline Uso do alimento como ferramenta pedagógica & 14 & 8,0 \\
\hline Distribuição de informativos, folders, textos ou gibis & 10 & 5,7 \\
\hline Oferta de alimentação saudável na escola & 4 & 1,9 \\
\hline Outras ações & 23 & 13,1 \\
\hline
\end{tabular}


Tabela 3. Associação entre a realização de ações de educação alimentar e nutricional, população escolar e características dos nutricionistas vinculados ao PNAE. Goiás, 2012.

\begin{tabular}{|c|c|c|c|c|c|}
\hline & \multicolumn{4}{|c|}{ Realiza ações de EAN } & \multirow{3}{*}{$\begin{array}{l}\text { Valor } \\
\text { de } p\end{array}$} \\
\hline & \multicolumn{2}{|c|}{ Sim } & \multicolumn{2}{|c|}{ Não } & \\
\hline & $\mathbf{n}$ & $\%$ & $\mathbf{n}$ & $\%$ & \\
\hline \multicolumn{6}{|l|}{ População escolar* $\left(\mathrm{n}=214^{* *}\right)$} \\
\hline $1^{\circ}$ Quartil & 51 & 26,2 & 3 & 15,8 & 0,554 \\
\hline $2^{\circ}$ Quartil & 49 & 25,1 & 4 & 21,1 & \\
\hline $3^{\circ}$ Quartil & 49 & 25,1 & 5 & 26,3 & \\
\hline $4^{\text {o }}$ Quartil & 46 & 23,6 & 7 & 36,8 & \\
\hline \multicolumn{6}{|c|}{ Presença de nutricionista RT $\left(\mathrm{n}=214^{* *}\right)$} \\
\hline Não & 10 & 5,1 & 5 & 26,3 & 0,001 \\
\hline Sim & 185 & 94,9 & 14 & 73,7 & \\
\hline \multicolumn{6}{|c|}{ Vínculo empregatício do nutricionista RT $\left(\mathrm{n}=178^{* * *}\right)$} \\
\hline Outro tipo de vínculo & 130 & 78,8 & 8 & 61,5 & 0,151 \\
\hline Concurso público & 35 & 21,2 & 5 & 38,5 & \\
\hline \multicolumn{6}{|c|}{ Frequência de atuação do nutricionista na alimentação escolar $\left(\mathrm{n}=178^{* * *}\right)$} \\
\hline Até 1 vez/semana & 42 & 25,5 & 4 & 30,8 & 0,948 \\
\hline 2 vezes/semana & 36 & 21,8 & 3 & 23,0 & \\
\hline 3 vezes/semana & 23 & 13,9 & 2 & 15,4 & \\
\hline 4 ou mais vezes/semana & 64 & 38,8 & 4 & 30,8 & \\
\hline \multicolumn{6}{|c|}{ Tempo de atuação do nutricionista na alimentação escolar do município $\left(\mathrm{n}=178^{* * *}\right)$} \\
\hline Até 1 ano & 56 & 33,9 & 7 & 53,8 & 0,680 \\
\hline 1 a 2 anos & 39 & 23,6 & 2 & 15,4 & \\
\hline 2 a 5 anos & 44 & 26,7 & 3 & 23,1 & \\
\hline 5 a 10 anos & 25 & 15,2 & 1 & 7,7 & \\
\hline$>10$ anos & 1 & 0,6 & 0 & 0,0 & \\
\hline \multicolumn{6}{|c|}{ Carga horária de trabalho do nutricionista $\left(\mathrm{n}=178^{* * *}\right)$} \\
\hline$<30$ horas/semana & 87 & 52,7 & 7 & 53,8 & 0,938 \\
\hline$\geq 30$ horas/semana & 78 & 47,3 & 6 & 46,2 & \\
\hline \multicolumn{6}{|c|}{ Dedicação do nutricionista à outras áreas, além da alimentação escolar $\left(\mathrm{n}=178^{* * *}\right)$} \\
\hline Não & 114 & 69,1 & 8 & 61,5 & 0,572 \\
\hline $\operatorname{Sim}$ & 51 & 30,9 & 5 & 38,5 & \\
\hline \multicolumn{6}{|c|}{ Nutricionista atua em outro município como RT do PNAE $\left(\mathrm{n}=178^{* * *}\right)$} \\
\hline Não & 121 & 73,3 & 10 & 76,9 & 0,777 \\
\hline Sim & 44 & 26,7 & 3 & 23,1 & \\
\hline \multicolumn{6}{|c|}{ Presença de outro(s) nutricionista(s) no QT do PNAE $\left(\mathrm{n}=178^{* *}\right)$} \\
\hline Não & 155 & 93,9 & 12 & 92,3 & \\
\hline $\operatorname{Sim}$ & 10 & 6,1 & 1 & 7,7 & 0,80 \\
\hline
\end{tabular}

${ }^{*}$ Fonte: Censo Escolar 2011 (Inep). ${ }^{* *}$ Todos os municípios do estudo. ${ }^{* *}$ Municípios em que o nutricionista foi entrevistado.

Neste sentido, mesmo entendendo que a EAN deve envolver outros atores sociais da comunidade escolar no que diz respeito às possibilidades de sucesso e mudanças comportamentais, no momento abordou-se o tema na perspectiva da atuação do nutricionista, frente às exigências contidas na Lei $n^{\circ} 11.947 / 2009$ e Resolução CD/ FNDE ${ }^{\circ} 26 / 2013^{2,3}$.

Como limitações observa-se que não foi identificado o tempo total de atuação do nutri- cionista junto ao PNAE, assim como o intervalo de tempo retrospectivo das informações prestadas sobre EAN.

Quanto à atuação do nutricionista, os resultados deste estudo evidenciaram que a maioria dos municípios estudados possuía nutricionista vinculado à alimentação escolar e que este profissional realizava ações de EAN nas escolas e creches. A frequência elevada de realização de ações de EAN corrobora com estudo do perfil 
do nutricionista do PNAE na região Nordeste do Brasil, onde dos 434 nutricionistas participantes, 80,7\% executavam ações de EAN junto aos estudantes ${ }^{11}$. No que se refere ao cenário nacional, em estudo realizado com 5.184 municípios brasileiros, 94,2\% afirmaram possuir nutricionista RT, e destes, apenas 37,9\% referiram desenvolver ações educativas de alimentação orgânica nas escolas ${ }^{12}$.

Estudo realizado em cidades do estado de Nova York (Estados Unidos) constatou que 83,0\% dos professores de escolas públicas de ensino primário trabalharam o tema alimentação e nutrição em sala de aula nos anos de 2007 e $2008^{13}$. Estimativas menores foram encontradas em escolas públicas de distritos da Califórnia (Estados Unidos), onde 36,0\% dos professores entrevistados mencionaram ensinar o tema $\mathrm{Nu}$ trição. Além disso, as horas de estudo sobre $\mathrm{Nu}$ trição eram menores no ensino médio quando comparado ao ensino primário ${ }^{14}$.

No presente estudo observou-se um maior percentual de municípios com ações de EAN somente nas escolas. Tal resultado pode ser explicado considerando os dados do Censo Escolar de 2011, ano anterior à concepção do estudo, que informava que o PNAE de muitos municípios goianos não atendia crianças em creches ${ }^{15}$.

A frequência com que as ações de EAN têm sido realizadas e a metodologia empregada possibilita um questionamento quanto à sua pertinência. A predominância de palestras e eventos em uma frequência semestral indica que o tema alimentação e nutrição pode não estar inserido de forma transversal no currículo escolar, como preconizam a Lei no 11.947/2009 e a Resolução CD/ FNDE no 26/2013. Apesar de mais da metade dos entrevistados ter afirmado que as ações de EAN estavam inseridas no PPP das escolas, grande parte não soube relatar em quais disciplinas isso ocorria. A inserção prioritária da EAN na disciplina de Ciências se assemelha a estudo realizado por Fiore et al. ${ }^{16}$, o qual constatou que temas de alimentação e nutrição apareciam com prioridade em Ciências, Geografia e História no material didático utilizado pela Secretaria de Educação do Estado de São Paulo; e por Watts et al. ${ }^{13}$, em que professores do estado de Nova York, nos Estados Unidos, relataram trabalhar estes temas nas aulas de educação física e ciências, prioritariamente.

Nesse contexto, Barbosa et al. ${ }^{17}$ consideram que a alimentação escolar é estratégia eficiente que deve ser inserida em temas relacionados à alimentação e nutrição para discussão no ambiente escolar. Sugere que todas as áreas do conhecimento podem contribuir e, embora não seja simples, o tema alimentação deve compor o projeto pedagógico das escolas de forma a garantir o trabalho transdisciplinar entre docentes e demais profissionais.

Em relação à coordenação e execução das ações de EAN, observou-se que o nutricionista RT atua predominantemente na coordenação e execução das ações, porém são citados também o professor, o coordenador pedagógico, o gestor local do PNAE ou o diretor das escolas. A multiprofissionalidade observada atende ao preconizado pela legislação do Programa ${ }^{3}$, e vai ao encontro do Marco de Referência de Educação Alimentar e Nutricional para as Políticas Públicas no Brasil ${ }^{18}$, documento construído coletivamente e publicado em 2012. A referida publicação orienta conceitos, princípios e diretrizes da EAN, defendendo uma prática educativa contínua e permanente, transdisciplinar, intersetorial e multiprofissional que visa promover a prática autônoma e voluntária de hábitos alimentares saudáveis.

Destaca-se, entretanto, o fato de membros do Conselho de Alimentação Escolar (CAE) terem sido citados como responsáveis pela coordenação e/ou execução das ações de EAN. Tais resultados podem indicar uma falha na compreensão das atribuições dos atores sociais envolvidos na execução do PNAE. De acordo com a Resolução CD/ FNDE no 26/2013, compete ao nutricionista RT a coordenação das ações de alimentação escolar, sendo atribuições do CAE o acompanhamento e a fiscalização dos recursos destinados à alimentação escolar, assim como o zelo pela qualidade dos alimentos e a emissão de parecer conclusivo acerca da aprovação da execução do Programa, não cabendo a este a coordenação ou a execução de ações educativas voltadas aos estudantes ${ }^{3}$.

A função dos demais atores sociais na realização das ações de EAN não se encontra descrita na legislação que regulamenta o PNAE, porém, de acordo com o CFN, a proposição de atividades educativas que envolvam a alimentação e a nutrição deve ser articulada pelo nutricionista, juntamente com a direção e a coordenação pedagógica das escolas ${ }^{9}$.

Nesse sentido, o desenvolvimento de projetos de EAN no ambiente escolar exige a colaboração mútua entre diretores, coordenadores, professores e nutricionistas ${ }^{7}$, sendo que a participação desse último em reuniões pedagógicas e em cursos de formação para professores pode apresentar resultados positivos quanto ao nível de informação desses atores da escola sobre alimentação e nutrição, potencializando, na sequência, o conhecimento dos estudantes sobre o tema ${ }^{19}$. 
É interessante observar que, neste estudo, as principais formas de orientação para incentivarem escolas e creches a realizarem EAN foram justamente estes espaços de interação. Acreditase que tanto a formação de professores pelos profissionais de saúde, quanto a intervenção direta junto aos escolares, apresentam o mesmo resultado quanto à produção e à construção do conhecimento sobre alimentação e nutrição pelos estudantes ${ }^{20,21}$.

Ao refletir sobre as atuais políticas públicas voltadas para a EAN, diante do estudo apresentado, verifica-se que existe uma crescente preocupação com as bases teórico-metodológicas, conforme também mostrado por Santos ${ }^{22}$.

Observou-se uma predominância de uso dos métodos de ensino tradicionais, tais como palestras e atividades não sequenciais executadas em eventos. A citação recorrente das palestras em atividades educativas realizadas por nutricionistas pode sinalizar uma postura prescritiva e verticalizada deste profissional, nem sempre intencional, mas que pode levar a uma sobreposição do saber científico em relação aos saberes populares dos grupos com os quais se envolve ${ }^{23}$. A EAN deveria propor a construção coletiva do conhecimento, amparada na problematização das vivências dos estudantes, não sendo, portanto pautada em práticas pedagógicas tradicionais e essencialmente teóricas ${ }^{24}$, e nesse escopo, o Marco de Referência de Educação Alimentar e Nutricional para as Políticas Públicas no Brasil faz essa orientação. Segundo esse documento, a EAN deve fazer uso de abordagens e recursos educacionais problematizadores e ativos que favoreçam o diálogo junto a indivíduos e grupos populacionais. Neste sentido, a abordagem da EAN precisa se estender para além da simples transmissão de conhecimentos, incluindo situações que promovam a reflexão sobre o cotidiano dos indivíduos com vistas à busca de soluções ${ }^{18}$.

A construção coletiva de uma agenda pública de EAN estabelecida pelo Marco sintetiza os esforços realizados para que a EAN seja efetivamente institucionalizada enquanto estratégia nas políticas públicas e nos diferentes espaços públicos. Para o alcance dessa prioridade, são necessários: planejamento e desenvolvimento de ações pautadas em evidências, promoção do diálogo com outras áreas de conhecimento e prática, ampliação de parcerias e compromissos, visibilidade das experiências bem sucedidas, investimentos em pesquisa sobre a temática bem como em metodologias e abordagens educativas, e sensibilização dos atores sociais envolvidos ${ }^{18}$.
Apesar das Diretrizes Curriculares Nacionais para os cursos de graduação em Nutrição, estabelecidas em $2001^{25}$, determinarem a formação de um profissional apto a atuar em políticas e programas de educação visando à promoção da saúde, os resultados deste estudo sinalizam para uma possível reavaliação no papel deste profissional como educador.

A ausência de teorias específicas de EAN faz com que se busque apoio nas teorias das Ciências Humanas e Sociais ${ }^{26}$ e até o momento não foram encontrados estudos específicos sobre como a EAN tem sido abordada nos currículos dos cursos de Nutrição no Brasil. Estudo realizado em 65 Instituições de Ensino Superior brasileiras que oferecem cursos de graduação em Nutrição constatou que as disciplinas que envolviam este campo do conhecimento, tais como 'Alimentação e Cultura', 'Antropologia da Alimentação' e 'Princípios da Comunicação' são oferecidas em apenas $24,6 \%, 44,6 \%$ e $32,3 \%$ dos cursos, respectivamente ${ }^{27}$.

Para o alcance de uma EAN inerente à preconizada pela legislação do $\mathrm{PNAE}^{3}$ e o Marco de Referência de Educação Alimentar e Nutricional para as Políticas Públicas no Brasil ${ }^{18}$, faz-se necessário investimentos na formação e na educação permanente, principalmente no que se refere a profissionais da comunidade escolar (gestores, coordenadores pedagógicos, professores, manipuladores de alimentos, nutricionistas ${ }^{24}$. Ressalta-se que o investimento na formação de nutricionistas implica não só na revisão dos componentes curriculares, como também na reflexão sobre o caminho da construção do conhecimento em nutrição, visto que a consolidação deste modelo de EAN demanda saberes transversais e interdisciplinares ${ }^{28}$.

Os resultados do presente estudo indicaram que a presença do nutricionista RT aumenta as chances de realização de ações de EAN nas escolas. Apesar de não apresentar significância estatística, municípios com maior população escolar apresentaram maior frequência de não realização das ações de EAN, o que pode estar relacionado ao fato da maioria dos municípios investigados apresentarem quantidade insuficiente de nutricionistas no QT do PNAE. Tal escassez pode dificultar a proposição de ações que sejam eficazes junto aos estudantes.

A ausência de outro(s) nutricionista(s) no QT do PNAE na maioria dos municípios indica uma falta de cumprimento da Resolução CFN no 465/2010. Chama atenção o caso de Goiás, onde apenas $29,0 \%$ dos municípios encontravam-se 
adequados numericamente ao parâmetro do Conselho Federal de Nutricionistas, o qual recomenda um nutricionista para até 500 estudantes. Em 26,2\% dos municípios seria necessário o acréscimo de mais um nutricionista no QT, por atenderem de 501 a 1000 estudantes, enquanto os demais deveriam contar com, no mínimo, mais dois nutricionistas no QT por apresentarem mais que 1000 estudantes. Ainda de acordo com o CFN, a recomendação do quantitativo de estudantes considera a dedicação do(s) nutricionista(s) durante 30 horas/semanais ${ }^{9}$, trazendo outro gargalo no atendimento do Programa, visto que a maioria dos nutricionistas entrevistados relatou carga horária semanal de trabalho inferior à regulamentada.

Os resultados encontrados são semelhantes a estudo realizado em 218 municípios do estado de Santa Catarina, onde 91,7\% contavam com um nutricionista no QT do PNAE. Em tal estudo, o percentual de inadequação do número de nutricionistas pela quantidade de alunos chegou a 97,4\% em municípios de médio e grande porte (população maior que 20.000 habitantes) e $63,4 \%$ naqueles de pequeno porte. $\mathrm{O}$ estudo considera ainda que 68,8\% dos municípios atendem mais que 500 alunos, sendo necessária a presença de outro(s) nutricionista(s) no QT do PNAE, além do $\mathrm{RT}^{29}$.

Outra similaridade com o estudo citado é o fato de que, assim como os nutricionistas em Goiás, a maioria daqueles atuantes no PNAE em Santa Catarina apresentou inconformidade quanto à carga horária destinada ao Programa. Cerca de 74,3\% dos nutricionistas tinham carga horária inferior a 80 horas mensais, o que não atendia a legislação do CFN vigente na época da coleta de dados (Resolução CFN no 358/2005), que preconizava 20 horas semanais de trabalho para escolas de período parcial ${ }^{30}$.

Ainda neste sentido, pesquisa desenvolvida com municípios brasileiros, observou que os locais onde o nutricionista possuía um tempo de atuação menor de 12 meses mostraram menores porcentagens de realização de ações educativas e inclusão do tema "alimentos orgânicos" no currículo escolar. Este resultado estava associado às condições de trabalho dos nutricionistas, onde mais da metade dos profissionais foram contratados para trabalhar menos de 30 horas por semana $^{12}$.

\section{Considerações finais}

A maioria dos entrevistados afirmou que são realizadas ações de EAN nas escolas e creches, porém com frequência semestral em grande parte dos municípios. Embora tenha sido afirmado que a EAN está inserida no PPP das escolas, poucos souberam relatar em quais disciplinas o tema se encontrava inserido.

O nutricionista parece estar envolvido em todas as fases, do planejamento à execução das ações de EAN. Porém as estratégias utilizadas são predominantemente pautadas em métodos tradicionais de ensino-aprendizagem, que nem sempre são eficientes na construção do conhecimento.

Investimentos governamentais são necessários para a formulação, a implementação e a avaliação de políticas públicas que incentivem a promoção e a manutenção da alimentação adequada e saudável no ambiente escolar. Ênfase deverá ser dada ao financiamento adequado na execução do Programa e na formação continuada dos atores envolvidos com a coordenação e a execução das ações de EAN.

Apesar das inegáveis conquistas com a inserção da EAN no PNAE, parece existir uma lacuna entre o discurso e a prática; entre o recomendado e o executado. Sugere-se a realização de estudos in loco, com vistas a avaliar a eficácia das metodologias empregadas; como tem sido a inserção do tema alimentação e nutrição nos currículos escolares, bem como o perfil do nutricionista para cumprir o proposto neste contexto. 


\section{Colaboradores}

Todos os autores revisaram e aprovaram o artigo, e os mesmos autorizam a reprodução do material pela Revista Ciência \& Saúde Coletiva. SU Silva participou da elaboração do projeto, da coleta e análise dos dados e redação do manuscrito. ET Monego e LM Sousa elaboraram o projeto, supervisionaram a coleta, contribuíram na análise dos dados e participaram da escrita do artigo. GM Almeida participou da coleta dos dados e revisão do manuscrito.

\section{Agradecimentos}

Às professoras Bethsáida Schmitz, Nilce Costa, Ida Menezes, Neila Machado, Maria Hermínia Domingues, Maria do Rosário Peixoto e Veruska Alexandre, pelas contribuições. Aos entrevistadores e à supervisora de campo Nicolly Gregório. Ao Fundo Nacional de Desenvolvimento da Educação do Ministério da Educação pelo financiamento.

\section{Referências}

1. Brasil. Decreto no 37.106, de 31 de março de 1955. Institui a Campanha de Merenda Escolar. Diário Oficial da União 1955; 2 abr.

2. Brasil. Presidência da República. Casa Civil. Lei $n^{\circ}$ 11.947, de 16 de junho de 2009. Dispõe sobre o atendimento da alimentação escolar e do Programa Dinheiro Direto na Escola aos alunos da educação básica; altera as Leis nos 10.880, de 9 de junho de 2004, 11.273, de 6 de fevereiro de 2006, 11.507, de 20 de julho de 2007; revoga dispositivos da Medida Provisória no 2.178-36, de 24 de agosto de 2001, e a Lei no 8.913, de 12 de julho de 1994; e dá outras providências. Diário Oficial da União 2009; 17 jun.

3. Brasil. Ministério da Educação. Fundo Nacional de Desenvolvimento da Educação. Resolução/CD/FNDE $n^{\circ} 26$, de 17 de junho de 2013. Dispõe sobre o atendimento da alimentação escolar aos alunos da educação básica no Programa Nacional de Alimentação Escolar - PNAE. Diário Oficial da União 2013; 18 jun.

4. Stefanini ML. Merenda escolar: história, evolução e contribuição no atendimento das necessidades nutricionais da criança [tese]. São Paulo: Universidade de São Paulo; 1997.

5. Brasil. Ministério da Educação. Fundo Nacional de Desenvolvimento da Educação. Resolução CD/FNDE no 32, de 10 de agosto de 2006. Estabelecer as normas para execução do Programa Nacional de Alimentação Escolar. Diário Oficial da União 2006; 25 ago.

6. Brasil. Portaria Interministerial no 1.010 , de 8 de maio de 2006. Institui as diretrizes para a promoção da alimentação saudável nas escolas de educação infantil, fundamental e nível médio das redes públicas e privadas, em âmbito nacional. Diário Oficial da União 2006; 9 maio.

7. Costa EQ, Ribeiro VB, Ribeiro ECO. Programa de alimentação escolar: espaço de aprendizagem e produção de conhecimento. Rev Nutr 2001; 14(3):225-229.

8. Brasil. Presidência da República. Casa Civil. Lei no 8.234, de 17 de setembro de 1991. Regulamenta a profissão de nutricionista e determina outras providências. Diário Oficial da União 1991; 18 set.

9. Conselho Federal de Nutricionistas. Resolução CFN no 465, de 23 de agosto de 2010. Dispõe sobre as atribuições do Nutricionista, estabelece parâmetros numéricos mínimos de referência no âmbito do Programa de Alimentação Escolar (PAE) e dá outras providências. Diário Oficial da União 2010; 25 ago.

10. Ação Fome Zero. Boletim de desempenho: prêmio gestor eficiente da merenda escolar 2012. São Paulo; 2012. [acessado 2013 Jan 08]. Disponível em: http://www. acaofomezero.org.br/premio/Paginas/home.aspx

11. Mello AL, Vidal Junior PO, Sampaio LR, Santos LAS, Freitas MCS, Fontes GAV. Perfil do nutricionista do Programa Nacional de Alimentação Escolar na região Nordeste do Brasil. Rev Nutr 2012; 25(1):119-132. 
12. Vieira AV, Corso ACT, González-Chica DA. Organic food-related educational actions developed by dieticians in Brazilian municipal schools. Rev Nutr 2014; 27(5):525-535.

13. Watts SO, Piñero DJ, Alter MM, Lancaster KJ. An Assessment of nutrition education in selected counties in New York State elementary schools. J Nutr Educ Behav 2012; 44(6):715-725.

14. Jones AM, Zidenberg-Cherr S. Exploring nutrition education resources and barriers, and nutrition knowledge in teachers in California. J Nutr Educ Behav 2015; 47(2):162-169.

15. Instituto Nacional de Estudos e Pesquisas Educacionais Anísio Teixeira [Internet]. Censo Escolar da Educação Básica de 2012. Brasília; 2012. [acessado 2013 Mar 28]. Disponível em: http://portal.inep.gov.br/basica-censo

16. Fiore EG, Jobstraibizer GA, Silva CS, Cervato-Mancuso AM. Abordagem dos temas alimentação e nutrição no material didático do ensino fundamental: interface com segurança alimentar e nutricional e parâmetros curriculares nacionais. Saúde Soc 2012; 21(4):10631074.

17. Barbosa NVS, Machado NMV, Soares MCV, Pinto ARR. Alimentação na escola e autonomia - desafios e possibilidades. Cien Saude Colet 2013; 18(4):937-945.

18. Brasil. Ministério do Desenvolvimento Social e Combate à Fome (MDS). Marco de referência de educação alimentar e nutricional para as políticas públicas. Brasília: MDS; 2012.

19. Detreagiachi CRP, Braga TMS. Projeto "criança saudável, educação dez": resultados com e sem intervenção do nutricionista. Rev Nutr 2011; 24(1):51-59.

20. Davanço GM, Taddei JAAC, Gaglianone CP. Conhecimentos, atitudes e práticas de professores de ciclo básico, expostos e não expostos a curso de educação nutricional. Rev Nutr 2004; 17(2):177-184.

21. Yokota RTC, Vasconcelos TF, Pinheiro ARO, Schmitz BAS, Coitinho DC, Rodrigues MLCF. Projeto "A escola promovendo hábitos alimentares saudáveis": comparação de duas estratégias de educação nutricional no Distrito Federal, Brasil. Rev Nutr 2010; 23(1):37-47.

22. Santos LAS. O fazer educação alimentar e nutricional: algumas contribuições para reflexão. Cien Saude Colet 2012; 17(2):453-462.

23. Caniné ES, Ribeiro VMB. A prática do nutricionista em escolas municipais do Rio de Janeiro: um espaço tempo -educativo. Ciênc Educ 2007; 13(1):47-70.

24. Bizzo MLG, Leder L. Educação nutricional nos parâmetros curriculares nacionais para o ensino fundamental. Rev Nutr 2005; 18(5):661-667.

25. Brasil. Ministério da Educação. Conselho Nacional de Educação. Resolução CNE/CES no 5, de 7 de novembro de 2001. Institui Diretrizes Curriculares Nacionais do Curso de Graduação em Nutrição. Diário Oficial União 2001; 9 nov.
26. Achterberg C, Miller C. Is one theory better than another in nutrition education? A viewpoint: more is better. J Nutr Educ Behav 2004; 36(1):40-43.

27. Recine E, Gomes RCF, Fagundes AA, Pinheiro ARO, Teixeira BA, Sousa JS, Toral N, Monteiro RAA. A formação em saúde pública nos cursos de graduação de nutrição no Brasil. Rev Nutr 2012; 25(1):21-33.

28. Santos LA. Avanços e desdobramentos do marco de referência da educação alimentar e nutricional para políticas públicas no âmbito na universidade e para os aspectos culturais da alimentação. Rev Nutr 2013; 26(5):595-600.

29. Machado PMO, Machado MS, Schmitz BAS, Corso ACT, Gonzalez-Chica DA, Vasconcelos FAG. Caracterização do Programa Nacional de Alimentação Escolar no estado de Santa Catarina. Rev Nutr 2013; 26(6):715725 .

30. Conselho Federal de Nutricionistas. Resolução CFN no 358/2005. Dispõe sobre as atribuições do Nutricionista no âmbito do Programa de Alimentação Escolar (PAE) e dá outras providências. Brasília; 2005. [acessado 2014 Out 07]. Disponível em: http://crn1.org.br/images/ pdf/res358.pdf

Artigo apresentado em 15/12/2015

Aprovado em 01/09/2016

Versão final apresentada em 03/09/2016 
